\title{
Potential neuroimaging biomarkers of pathologic brain changes in Mild Cognitive Impairment and Alzheimer's disease: a systematic review
}

\author{
Qingwei Ruan', Grazia D'Onofrio ${ }^{2^{*}}$, Daniele Sancarlo², Zhijun Bao ${ }^{1}$, Antonio Greco ${ }^{2}$ and Zhuowei Yu${ }^{1,3^{*}}$
}

\begin{abstract}
Background: Neuroimaging-biomarkers of Mild Cognitive Impairment (MCl) allow an early diagnosis in preclinical stages of Alzheimer's disease (AD). The goal in this paper was to review of biomarkers for Mild Cognitive Impairment (MCI) and Alzheimer's disease (AD), with emphasis on neuroimaging biomarkers.

Methods: A systematic review was conducted from existing literature that draws on markers and evidence for new measurement techniques of neuroimaging in $A D, M C l$ and non-demented subjects. Selection criteria included: 1) age $\geq 60$ years; 2) diagnosis of AD according to NIAAA criteria, 3) diagnosis of $\mathrm{MCl}$ according to NIAAA criteria with a confirmed progression to AD assessed by clinical follow-up, and 4) acceptable clinical measures of cognitive impairment, disability, quality of life, and global clinical assessments.

Results: Seventy-two articles were included in the review. With the development of new radioligands of neuroimaging, today it is possible to measure different aspects of $A D$ neuropathology, early diagnosis of $\mathrm{MCl}$ and $A D$ become probable from preclinical stage of $A D$ to $A D$ dementia and non-AD dementia.

Conclusions: The panel of noninvasive neuroimaging-biomarkers reviewed provides a set methods to measure brain structural and functional pathophysiological changes in vivo, which are closely associated with preclinical AD, $\mathrm{MCl}$ and non-AD dementia. The dynamic measures of these imaging biomarkers are used to predict the disease progression in the early stages and improve the assessment of therapeutic efficacy in these diseases in future clinical trials.
\end{abstract}

Keywords: Alzheimer's disease, Mild cognitive impairment, Magnetic resonance imaging, Positron emission tomography

\footnotetext{
* Correspondence: g.donofrio@operapadrepio.it; hdyuzhouwei@163.com ${ }^{2}$ Geriatric Unit \& Laboratory of Gerontology and Geriatrics, Department of Medical Sciences, IRCCS "Casa Sollievo della Sofferenza", San Giovanni Rotondo, Foggia, Italy

${ }^{1}$ Shanghai Institute of Geriatrics and Gerontology, Shanghai Key Laboratory of Clinical Geriatrics, Department of Geriatrics, Huadong Hospital, and Research Center of Aging and Medicine, Shanghai Medical College, Fudan University, Shanghai 200040, China

Full list of author information is available at the end of the article
} 


\section{Background}

Alzheimer's disease (AD) is the most common agerelated neurodegenerative disease, characterized by progressive cognitive decline, accounts for $50-75 \%$ of the global dementia population, with a greater proportion in the higher age ranges [1].

Clinicians and researchers have recently updated the AD diagnostic criteria for use in clinical practice and research [2]. Biomarkers (e.g., CSF protein levels, neuroimaging) may be used to rule out other causes of dementia (e.g., vascular) and to support the AD diagnosis in cases with unclear or atypical presentations. Attempts to diagnose $\mathrm{AD}$ at an earlier stage have led to the appearance of new medical terminologies such as pre-clinical $\mathrm{AD}$, prodromal $\mathrm{AD}$ or mild cognitive impairment (MCI). Recently, new criteria for diagnosis of MCI in clinical and research settings have been published $[3,4]$.

In this context neuroimaging and fluid biomarkers for amyloid deposition and hippocampal atrophy can be measured more than 10 years before the onset of dementia [5, 6]. The application of these markers could enhance the specificity of clinical diagnosis and improve the prediction of the disease progression. The objective in this paper was to review of biomarkers for Mild Cognitive Impairment (MCI) and $\mathrm{AD}$, with emphasis on neuroimaging biomarkers.

\section{Methods}

The search strategy and analysis was informed by: the study's aims, previous systematic reviews using qualitative data, and best practice recommendations in the research literature [7, 8]. Literature searches were conducted over MEDLINE (2000 to June 2015) and Pubmed (2000 to June 2015), using the OVID search interface. The searches were limited to human studies in English language including potential Magnetic Resonance Imaging (MRI) and Positron Emission Tomography (PET) biomarkers.

\section{Study selection}

A single reviewer examined the abstracts retrieved by the electronic search in order to identify articles that met the inclusion criteria and to be fully reviewed.

Inclusion criteria: 1 ) age $\geq 60$ years; 2 ) diagnosis of $\mathrm{AD}$ according to the National Institute on AgingAlzheimer's Association (NIAAA) criteria [2], 3) diagnosis of MCI according to NIAAA criteria [4] with a confirmed progression to AD assessed by clinical follow-up, and 4) acceptable clinical measures of cognitive impairment, disability, quality of life, and global clinical assessments.

Exclusion criteria: 1) no English editing (as we lacked resources for translation), 2) diagnosis of non-AD dementia, and 3) MCI not progressed in AD.

\section{Data extraction}

In total, 2243 articles, reports and reviews were identified. After reviewing abstracts, 425 were excluded on the basis of the aforementioned inclusion/exclusion criteria and the removal of duplicates (n. 1595). A further 151 were excluded after more in-depth examination (on the basis of the same inclusion/exclusion criteria). Thus, 72 published studies were eligible for the current review (Fig. 1).

Data extraction followed a number of stages. After preliminary categorisation, categories were divided among the authors according to expertise. Each category was reviewed in depth by at least one author and the lead author:

Potential structural neuroimaging biomarkers

Functional neuroimaging biomarkers

Co-authors provided a detailed summary of each study including its strengths and weaknesses, as well as an overall appraisal of the category [9, 10]. Quality of study reporting was assessed using the Standards for the Reporting of Diagnostic accuracy studies in dementia (STARDdem) [11].

\section{Results and discussions}

Potential structural neuroimaging biomarkers Structural MRI (sMRI)

Medial temporal and hippocampal atrophy were the most common structural MRI (sMRI) markers of progression to $\mathrm{AD}[12,13]$. The 3-dimensional patterns of cerebral atrophy progression over multiple serial MRI were consistent with neurofibrillary pathological staging scheme in $\mathrm{AD}$, showing that the earliest changes in the anterior medial temporal lobe and fusiform gyrus occur at least 3 years before conversion to AD [14]. However, one study showed that both baseline enthorhinal volume and its slope of decline, but not initial hippocampal size and its rate of decline, were independent predictors of incident AD [15].

Molecular biomarkers of neuronal injury that are present in advance of atrophy offer a complementary target for sMRI [16]. Model-based magnetization transfer (mMT) imaging could improve classification of subjects with early $\mathrm{AD}$ and MCI compared with magnetization transfer ratio (MTR) [17].

Individual biomarkers, such as white matter hyperintensities (WMH), cerebral volume, hippocampal volume, entorhinal cortex thickness, ventricle volume, and CSF markers had limited predictive value for cognitive decline [18]. Combination of sMRI and plasma biomarkers, such as whole genome single nucleotide polymorphism data, tocopherols and tocotrienols, or CSF total tau, phosphorylated tau (p-tau) and $A \beta 42$ : amyloid- $\beta 42$ (A $\beta 42)$ could 


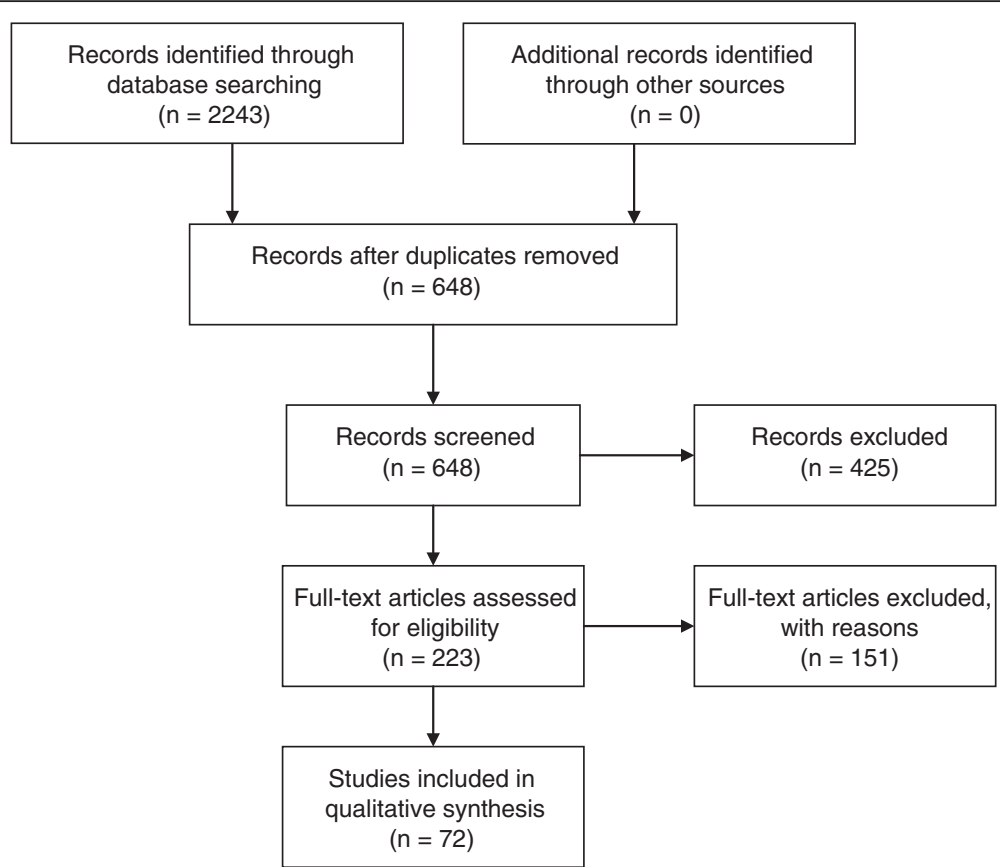

Fig. 1 Flow diagram outlining the selection procedure to identify articles which were included in the systematic review of neuroimaging biomarkers of pathologic brain changes in Mild Cognitive Impairment and Alzheimer's disease

enhance the accurancy of differentiating subjects with $\mathrm{AD}$ and MCI from cognitively normal subjects [19-21]. Annual ventricular volume changes in serial sMRI were related to concurrent change on general cognitive and functional indices in amnestic MCI (aMCI) and $\mathrm{AD}$, and were influenced by Apolipoprotein E (ApoE) genotype [22]. The combination of imaging and CSF biomarkers could improve the conversion prediction from MCI to AD $[21,23-25]$ and time to develop dementia in subjects with MCI and amyloid pathology [26]. Baseline fludeoxyglucose (FDG) Positron Emission Tomography (PET) added the greatest prognostic information compared with baseline clinical testing [24], but marginally predicted longitudinal cognitive decline [25]. Baseline sMRI was slightly better predictor of future clinical/functional decline than CSF t-tau/A 42 [21]. MRI imaging might be a more practical clinical biomarker for early detection of AD [27]. Multiple MRI markers of underlying dementia pathologies improve the ability to identify patients with prodromal dementia over a single MRI marker [28]. The areas of hypoperfusion in patients with asymmetric cortical degenerative syndromes measured by single-photon emission computed tomography (SPECT) were larger than the corresponding atrophic regions showed by sMRI [29]. The combination of both was useful in identifying the regional structural and functional cerebral abnormalities.

The mild or severe atropy in sMRI with severe decline in parietal regional Cerebral blood flow (CBF) on SPECT could distinguish AD from FTD and $\mathrm{VaD}$ patients [30].
The most common non-AD dementia is vascular dementia $(\mathrm{VaD})$ with considerable overlap with $\mathrm{AD}$ pathology. $\mathrm{VaD}$ results from cerebrovascular and cardiovascular disorders. Non-significant vascular lesions on structre brain imaging result in overdiagnosis of $\mathrm{VaD}$ [31]. A significantly higher allele frequency of ApoEs4 in $\mathrm{AD}$ patients was found than these with $\mathrm{VaD}$ in a community population [32]. The increase of WMH in deep white matter, but not WMH in periventricular white matter on MRI scans indicates $86 \%$ classification accuracy [33]. MRI measure of infarcts, a vascular brain injury, were significantly related to medial-temporal and cerebral atropy in $\mathrm{VaD}$ but not in AD patients with $71 \%$ sensitivity and $76 \%$ specificity [34]. The combination of MRI and SPECT could differentiate $\mathrm{VaD}$ with sensitivity $88 \%$, specificity $75 \%$ and FTD with sensitivity $73 \%$, specificity $78 \%$ respectively, and enhance specificity of AD diagnosis by CSF biomarkers from 71 to $93 \%$ [35].

\section{Diffusion MRI}

The diffusion tensor imaging (DTI) metrics including fractional anisotropy (FA), mean diffusivity, axial diffusivity and radial diffusivity (RA), can assess the connectivity between brain regions [36-38]. Both vascular and $\mathrm{AD}$ degenerative process have different region-specific structural injury patterns of cerebral white matter [39]. The combination of mean tanscallosal prefrontal FA and the Fazekas score in volume by T2-weighted measures 
could differentiate VaD from AD with $87.5 \%$ accuracy, $100 \%$ specifity and $93 \%$ sensitivity [40].

The reduced FA and RA values in patients with $\mathrm{AD}$ than in patients with MCI suggested that the brain DTI was useful to confirm clinical manifestation of $\mathrm{AD}$ but not in the detection of MCI [41]. White matter microstructural changes identified with DTI derived FA doesn't appear as a significant predictor of cognition [42]. In another study, it was shown that high WMH predict progression from normal to $\mathrm{MCI}$, but not progression from MCI to dementia [43]. Conversely, the brain parenchymal fraction (BPF) which showed wholebrain atrophy, did not predict conversion from normal to MCI but predicted conversion to dementia [43]. In a study, it was shown that posterior periventricular and corpus callosum extension of WMH associated with $\mathrm{MCI}$ and $\mathrm{AD}$ indicate involvement of strategic white matter bundles that may contribute to the cognitive deficits [44]. Increased amyloid burden, as measured with florbetapir PET imaging, was associated with changes in weighted structural connectome metrics independent of brain region [45]. The decrease of FA and the mode of anisotropy in intracortically projecting fiber tracts of MCI-AD and AD subjects suggested early axonal degeneration in intracortical projecting fiber tracts [46]. To assess the decrease of the cingulum fibers using DTIderived FA could help early diagnosis of AD [47]. The hippocampal apparent diffusion coefficients were higher in MCI and AD subjects than in controls [48]. The elevated apparent diffusion coefficients in hippocampus may indicate early pre-clinical AD.

\section{Functional neuroimaging biomarkers PET imaging for metabolism status}

A study showed that $30 \mathrm{~min}$ was enough foroptimal FDG accumulation for AD PET scan, especially for the early stage of $\mathrm{AD}$ [49]. Bilateral temporoparietal hypometabolism on [18 F] fludeoxyglucose-positron emission tomography (FDG-PET) could differentiate AD dementia from $\mathrm{VaD}$ dementia with leukoaraiosis [50]. (18)FDG-PET showed FDG uptake reductions in AD related brain regions in cognitively normal individual with ApoE\&4 genotype and subjective cognitive complaint [51], or a maternal history of AD [52]. Compared with nonconverters, converters of MCI patients had lower FDG uptake in the right temporoparietal contex [53]. Hypometabolism in brain regions may be related to ongoing pathologies and to reduced neuronal input in remote but functionally connected regions [54]. MCI patients who did not develop dementia after 2 years showed even higher uptake in the basal forebrain than those who developed dementia [55]. Longitudinal changes in individual subjects in the spatial pattern of brain glucose metabolism measured with (18)F-FDG
PET showed correlations with the cognitive decline of $\mathrm{AD}$ and $\mathrm{MCI}[56,57]$.

The combination C-labeled Pittsburgh Compound-B $([(11) \mathrm{C}] \mathrm{PIB})$ and FDG track molecular changes could show different stages of AD. Increased [(11)C]PIB images of binding potential was found in MCI patients and progressive decrease of FDG uptake was only observed in AD patients [58]. The hypoperfusion patterns showed by arterial spin labeling MRI and hypometabolism patterns showed by FDG-PET could provide largely overlapping information of fuctional deficits in affected areas of AD [59]. Regional cerebral blood flow measured by [11C]dihydrotetrabenazine (DTBZ)-PET also provided similar information in assessment of regional cerebral metabolic deficits in mild dementia and MCI with FDGPET, and exhibited a mild decrease in sensitivity [60]. Preclinical and clinical studies have shown that the use of PET imaging for tracking neuroinflammatory changes seems to have a promising role in AD and other central nervous system (CNS) pathologies [61, 62]. Translocator protein (TSPO) radioligands [11C](R)-PK11195, [11C]DAA1106 and [11C]PBR28 suggest the increased expression of TSPO by activated microglia in AD patients [62].

The radioligand 11C-deuterium-L-deprenyl ([11C]DED) showed the highest binding among PIB in MCI individuals, that suggests the reactive astrocytosis occurs at the early stages of $\mathrm{AD}$ [63]. Activation of cytosolic phospholipase A2 (cPLA2) and secretory phospholipase A2 (sPLA2) after microglia-derived inflammatory cytokines bind astrocytic cytokine receptors results in the hydrolysis of membrane phospholipids, liberating arachidonic acid (AA) [64]. A common single nucleotide polymorphism (rs6971) in exon 4 of the TSPO gene has been identified as the key determinant of affinity with second generation TSPO radioligands [11C]DAA1106 and [11C]PBR28 [65]. Cerebral blood flow, which is reduced in $\mathrm{AD}$, highly influences monoamine oxidase $\mathrm{B}$ (MAO-B) binding that seems to increase with age in almost all brain regions (with the exception of the cingulate gyrus) in healthy human subjects [62]. PET neuroinflammation imaging may, alternately, be more useful in monitoring the responsivity to antiinflammatory therapies in AD.

\section{PET imaging for amyloid load}

Amyloid accumulation evidenced by florbetapir PET may be a potential marker of preclinical AD. Cognitively normal subjects with florbetapir uptake increase in brain were associated with worse globe cognitive performance [66]. [(11)C]PIB data showed expected differences among subjects of control, MCI and AD [67-69] and identified subjects with significant annual increases in amyloid load across the subject groups [67]. 
Longitudinal studies showed that PET Aßpositive subjects of cognitively normal, $\mathrm{MCI}$ and $\mathrm{AD}$ subjects had greater cognitive and global deterioration than $\mathrm{A} \beta$ negative subjects $[70,71]$ and $A \beta$ positive subjects of $\mathrm{MCI}$ had higher risk for conversion to $\mathrm{AD}$ than $\mathrm{A} \beta$ negative subjects [71, 72]. Florbetapir PET measurements showed that $76 \% \mathrm{AD}, 38 \% \mathrm{MCI}$, and $14 \%$ cognitively normal subjects was amyloid positive [73]. The global cortex standardized uptake value ratio of Florbetapir could differentiate $\mathrm{AD}$ and $\mathrm{MCI}$ from healthy normal controls with high specificity and sensitivity [74]. Amyloid deposition has already slowed or ceased when dementia occurs [75]. Combination baseline sMRI and (18)F-florbetaben (FBB) uptake values did not improve predictive accuracy of MCI conversion to AD [76]. CSF A $\beta 42$ did not always become abnormal prior to fibrillar $A \beta$ accumulation early in the course of disease [77]. 2-(1-\{6-[(2fluorine 18-labeled fluoroethyl)methylamino]-2-napthyl\}ethylidene) malononitrile ([(18)F]FDDNP) provides a measure of both amyloid and tau. [(18)F]FDDNP could predict future cognitive decline and conversion of $\mathrm{MCI}$ to $\mathrm{AD}[78]$.

\section{PET radioligands of tau protein}

In the amyloid cascade hypothesis, although tau pathology is considered secondary to A $\beta$ pathology, the postmortem classification of AD cases into pathological subtypes with distinct clinical characteristics is determined by the localization and distribution of tau pathology in the brain $[79,80]$. Abnormal burden of tau species can accurately predict disease severity and the rate of cognitive decline $[79,80]$. The recent progress in tau protein ligands makes tau PET imaging a potential biomarker. Radioligands [18 F]-T807 and [18 F]-T808 used in human brain images with different pharmacokinetic characteristics were the most selective compounds for filamentous tau, and the level of selective binding with tau was 27-fold higher than that of $\mathrm{A} \beta[81,82]$. A phenyl/pyridinyl-butadienyl-benzothiazoles/benzothiazolium (PBB) showed the greatest specificity for tau in vivo PET imaging of tau transgenic mouse models, and [(11)C]PBB3 patterns were consistent with the spreading of tau pathology with $\mathrm{AD}$ progression and non-AD tauopathy corticobasal syndrome in a clinical PET study [83].

\section{fMRI and SPECT}

A set of medial prefrontal and temporo-parietal regions, such as the posterior cingulate and hippocampus, are referred to as the default-mode network (DMN), which is most active at rest and deactivated during cognitive tasks. Amyloid deposition in $\mathrm{AD}$ brains is most obvious in brain areas of the default network. Functional MRI showed that the DMN exhibits both reduced functional connectivity and impaired task-induced deactivation in $\mathrm{AD}$, mild cognitive impairment (MCI) and pre-MCI [84-89].

Subjects with aMCI showed decreased DMN activity in memory function related brain regions, such as left medial temporal lobe before atrophy was detectable by sMRI [86]. Based on a multi-modal imaging approach, including FDG-PET and sMRI and diffusion-weighted MRI results, $\mathrm{AD}$ patients revealed decreased structural and functional connections, corresponding consistent reduction of metabolic activity and atrophy within DMN [90]. The disrupted connectivity in $\mathrm{AD}$ turns the high metabolic activity of DMN into hypometabolism [84]. A 2- to 3- year follow-up study revealed that functional connectivity indices could predict conversion of MCI to AD [91]. Education, a factor in cognitively demanding tasks, reduces AD risk by reducing neuronal activity and $A \beta$ generation within the default network [92]. It was observed that in the earliest phase of MCI the individuals exhibited significantly greater hippocampal activation than controls even if two group without difference in hippocampal or entorhinal volumes [93]. Longitudinal fMRI in cognitively normal elderly participants reveals that subjects with the highest hippocampal activation at baseline and the greatest loss of hippocampal activation demonstrated more rapid cognition decline [94]. Compensatory efforts as a result of preclinical pathological changes in learning and memory tasks induce enhanced brain activity in some areas in the initial targets of $\mathrm{AD}$, and these changes may precede the diagnosis of AD by 30 years [95]. Neuronal activity stimulates aerobic glycolysis and increases $A \beta$ production and secretion into the interstitial fluid (ISF). The degree of $A \beta$ aggregation and plaque deposition is directly proportional to ISF $A \beta$ concentrations in vivo [96]. Factors including elevated endogenous neuronal activity may accelerate the $\mathrm{A} \beta$ deposition process. Cognitively normal ApoE\&4 carriers show elevated resting-state activity in the default network and increased hippocampal activation in fMRI during a memory-encoding task compared to non-carriers [97]. Cerebral perfusion abnormalities were evident in $A D$ progression. CBF was a more sensitive parameter than cerebral blood volume for perfusion normalities and appeared before the latter in the progression of $\mathrm{AD}$ [98]. A reduced $\mathrm{CBF}$ of the left posterior cingulated gyrus [99], bilateral prefrontal and frontal, and left parietal [100], and right parietal and hippocampal regions [101] evidenced by SPECT could predict the conversion of $\mathrm{MCI}$ to $\mathrm{AD}$ at least 2 years before clinical AD. But low parietal and medial temporal flow using SPECT demonstrated limited utility in predicting MCI conversion to AD [102]. Semiquantitative circumferential-profile analysis of brain SPECT showed that AD patients have more significant reductions in the posterior temporo-parietal regions, and white matter $\mathrm{VaD}$ patients have greater reducutions in the frontal brain regions [103]. 
Combination the pattern of hypoperfusion and the severity of memory deficits could predict the risk of progression to $\mathrm{AD}$ in $\mathrm{MCI}$ subjects with a sensitivity and specificity $[102,104]$.

Regional CBF measured by Arterial spin-labeling (ASL) magnetic resonance showed that regional CBF of AD patients was significantly lower in both the bilateral frontal and temporal lobes, and the value of $\mathrm{VaD}$ patients was significantly in left frontal and temporal white matter [105]. SPECT imaging of regional benzodiazepine receptors (rBZR) can reflect neuronal integrity in the cerebral cortex. $\mathrm{VaD}$ and mixed $\mathrm{AD} / \mathrm{VaD}$ dementia showed predominant reduction of regional $\mathrm{CBF}$ and rBZR in the frontal lobe, and $\mathrm{AD}$ in parietotemporal lobe. Furthermore, rBZR images of $\mathrm{VaD}$ and mixed dementia showed more extensive and severe defects than CBF images, and $\mathrm{CBF}$ images of $\mathrm{AD}$ showed more extensive defects than rBZR [106]. Although the heterogeneity index of the whole brain CBF on SPECT images was not significantly different between the $\mathrm{AD}$ and $\mathrm{VaD}$ groups, the herogeneity of $\mathrm{CBF}$ for $\mathrm{AD}$ and $\mathrm{VaD}$ was posterior and anterior-dominant respectively [107].

\section{Conclusion}

The use in clinical practice of neuroimaging biomarkers of brain pathological processes could permit to perform an early diagnosis and to estimate the disease progression. Some neuroimaging-biomarkers have been widely used in clinical diagnosis of AD. The more neuroimaging biomarkers are still used for clinical studies. The use of neuroimaging biomarkers depended on the stage of disease progress. The proposed pathophysiological sequence contains $A \beta$ accumulation, neuronal dysfunction (synaptic dysfunction, glial activation, tangle formation), brain atrophy due to neuronal death, and finally cognitive impairment [108].

Amyloid imaging is useful for differential diagnosis in early-onset dementia and clinical diagnosis of $\mathrm{AD}$ in noncarriers of ApoEe4 who are older than 70 years [5]. However, amyloid imaging may not be sufficient to make correct diagnosis in an individual [109]. PET with FDG, tau and other neurochemical tracers, fMRI and SPECT are used to measure the neuronal dysfunction. Finally, sMRI, advanced MRI techniques, such as DTI and MRS are used to show atrophy and hypoperfusion in cortex and white matter. The ordering and the sigmoidal-like time changes of imaging biomarkers are useful to detect early pathophysiological changes in preclinical stage; the extensive and severe impairment means greater AD-like pathology. Therefore, imaging biomarkers often are used to detect the comversion of preclinical $\mathrm{AD}$ and $\mathrm{MCI}$ to $\mathrm{AD}$ and predict outcomes of clinical intervention trials. Particularly, imaging biomarkers are helpful to differentiate $\mathrm{AD}$ dementia from $\mathrm{VaD}$ and other non- $\mathrm{AD}$ dementia. To find early different vascular pathological contribution to $\mathrm{AD}$ and $\mathrm{VaD}$, an advanced dynamic contrast-enhanced MRI could quantify hippocampus blood brain barrier permeability in the living human brain; CA1 and dentate gyrus subdivisions showed obviously worsened in patients with mild cognitive impairment,which was correlated with injury to BBBassociated pericytes [110].

The combination of structural, functional neuroimaging and fluid biomarkers improved the accuracy of prediction [111]. With same diagnosis power, one should give preference to the less expensive, safer and less invasive techniques. However, some factors, including environmental factor, ApoE genetic variation and brain or cognitive reserve can affect or alter the cuves of these biomarkers.

\section{Ethics approval and consent to participate}

Not applicable.

\section{Consent for publication}

Not applicable.

\section{Availability of data and materials \\ Not applicable.}

\section{Abbreviations}

[(11)C]PIB: C-labeled Pittsburgh Compound-B; [(18)F]FDDNP: 2-(1-\{6-[(2-

fluorine 18-labeled fluoroethyl)methylamino]-2-napthyl\}ethylidene) malononitrile; [11C]-DED: 11C-deuterium-L-deprenyl; AA: arachidonic acid;

AD: Alzheimer's disease; ApoE: apolipoprotein E; A 342 : amyloid- $\beta 42 ;$ BPF: brain parenchymal fraction; CBF: cerebral blood flow; CNS: central nervous system; CPLA2: cytosolic phospholipase A2; CSF: cerebrospinal fluid; DMN: default-mode network; DTBZ: [11C]dihydrotetrabenazine; DTI: diffusion tensor imaging; FA: fractional anisotropy; FBB: (18)F-florbetaben;

FDG: fludeoxyglucose; ISF: interstitial fluid; MAO-B: monoamine oxidase B; MCl: mild cognitive impairment; mMT: model-based magnetization transfer; MRI: magnetic resonance imaging; MTR: magnetization transfer ratio; NIAAA: National Institute on Aging-Alzheimer's Association; PBB: phenyl/ pyridinyl-butadienyl-benzothiazoles/benzothiazolium; PET: positron emission tomography; p-tau: phosphorylated tau; RA: radial diffusivity; rBZR: regional benzodiazepine receptors; rs6971: single nucleotide polymorphism; SMRI: structural MRI; SPECT: single-photon emission computed tomography; SPLA2: secretory phospholipase A2; STARDdem: Standards for the Reporting of Diagnostic accuracy studies in dementia; TSPO: translocator protein; VaD: vascular dementia; WMH: white matter hyperintensities.

\section{Competing interests}

The authors declare that they have no competing interests.

\section{Authors' contributions}

$\mathrm{QR}$ and GD conceived of the study. QR, ZB and ZY created the search protocol. QR examined abstracts retrieved. QR and GD contributed to manuscript preparation. DS, AG, ZB and ZY contributed to analysis, drafting the paper and the conclusions. The authors confirm that the manuscript has been read and approved by all named authors and that there are no other persons who satisfied the criteria for authorship but are not listed. The authors further confirm that the order of authors listed in the manuscript has been approved by all. 


\section{Acknowledgments}

This study was supported by the Shanghai Hospital Development Center (No. SHDC12014221) and Shanghai Key Laboratory of Clinical Geriatric Medicine (13dz2260700)

\section{Funding}

The authors did not receive grants or outside funding in support of their research for or preparation of this manuscript.

\section{Author details}

'Shanghai Institute of Geriatrics and Gerontology, Shanghai Key Laboratory of Clinical Geriatrics, Department of Geriatrics, Huadong Hospital, and Research Center of Aging and Medicine, Shanghai Medical College, Fudan University, Shanghai 200040, China. ${ }^{2}$ Geriatric Unit \& Laboratory of Gerontology and Geriatrics, Department of Medical Sciences, IRCCS "Casa Sollievo della Sofferenza", San Giovanni Rotondo, Foggia, Italy. ${ }^{3}$ Huadong Hospital, Shanghai Medical College, Fudan University, 221 West Yan An Road, Shanghai 200040, P.R. China.

\section{Received: 27 January 2016 Accepted: 9 May 2016}

\section{Published online: 16 May 2016}

\section{References}

1. Braak H, Del Tredici K. Where, when, and in what form does sporadic Alzheimer disease begin? Curr Opin Neurol. 2012;25(6):708-14.

2. McKhann GM, Knopman DS, Chertkow H, et al. The diagnosis of dementia due to Alzheimer's disease: recommendations from the National Institute on Aging-Alzheimer's Association workgroups on diagnostic guidelines for Alzheimer's disease. Alzheimers Dement. 2011;7:263-9.

3. Petersen RC, Smith GE, Waring SC, et al. Mild cognitive impairment: clinical characterization and outcome. Arch Neurol. 1999:56:303-8.

4. Albert MS, DeKosky ST, Dickson D, et al. The diagnosis of mild cognitive impairment due to Alzheimer's disease: recommendations from the National Institute on Aging-Alzheimer's Association workgroups on diagnostic guidelines for Alzheimer's disease. Alzheimers Dement. 2011;7: 270-9.

5. Jansen WJ, Ossenkoppele MR, Knol DL, et al. Prevalence of cerebral amyloid pathology in persons without dementia A meta-analysis. JAMA. 2015; 313(19):1924-38.

6. Villemagne $V L$, Burnham $S$, Bourgeat $P$, et al. Amyloid $\beta$ deposition, neurodegeneration, and cognitive decline in sporadic Alzheimer's disease: A prospective cohort study. Lancet Neurol. 2013;12(4):357-67.

7. Dixon-Woods M, Agarwal S, Jones D, et al. Synthesising qualitative and quantitative evidence: a review of possible methods. J Health Serv Res Policy. 2005;10:45-53.

8. Hannes K, Macaitis K. A move to more systematic and transparent approaches in qualitative evidence synthesis: update on a review of published papers. Qual Res. 2012:12:402-42.

9. Barnett-Page $E$, Thomas J. Methods for the synthesis of qualitative research: a critical review. BMC Med Res Methodol. 2009:9:59.

10. Grant MJ, Booth A. A typology of reviews: an analysis of 14 review types and associated methodologies. Health Info Libr J. 2009:26:91-108.

11. Noel-Storr AH, McCleery JM, Richard E, et al. Reporting standards for studies of diagnostic test accuracy in dementia: The STARDdem Initiative. Neurology. 2014:83(4):364-73.

12. Jack Jr CR, Petersen RC, Xu YC, et al. Prediction of AD with MRI-based hippocampal volume in mild cognitive impairment. Neurology. 1999;52(7): 1397-403.

13. Risacher SL, Saykin AJ, West JD, et al. Baseline MRI predictors of conversion from $\mathrm{MCl}$ to probable AD in the ADNI cohort. Curr Alzheimer Res. 2009;6(4): 347-61.

14. Whitwell JL, Przybelski SA, Weigand SD, et al. 3D maps from multiple MRI illustrate changing atrophy patterns as subjects progress from mild cognitive impairment to Alzheimer's disease. Brain. 2007;130(Pt 7):1777-86

15. Stoub TR, Bulgakova M, Leurgans $S$, et al. MRI predictors of risk of incident Alzheimer disease: a longitudinal study. Neurology. 2005:64(9):1520-4.

16. Jack Jr CR. Alzheimer disease: new concepts on its neurobiology and the clinical role imaging will play. Radiology. 2012;263:344-61.

17. Wiest $R$, Burren $Y$, Hauf $M$, et al. Classification of mild cognitive impairment and Alzheimer disease using model-based MR and magnetization transfer imaging. Am J Neuroradiol. 2013;34(4):740-6.
18. Nettiksimmons J, Harvey D, Brewer J, et al. Subtypes based on cerebrospinal fluid and magnetic resonance imaging markers in normal elderly predict cognitive decline. Neurobiol Aging. 2010;31(8):1419-28.

19. Kong D, Giovanello KS, Wang Y, et al. Predicting Alzheimer's Disease Using Combined Imaging-Whole Genome SNP Data. J Alzheimers Dis. 2015:46(3):695-702

20. Mangialasche F, Westman E, Kivipelto M, et al. Classification and prediction of clinical diagnosis of Alzheimer's disease based on MRI and plasma measures of a- $/ \gamma$-tocotrienols and $\gamma$-tocopherol. J Intern Med. 2013;273(6):602-21.

21. Vemuri P, Wiste HJ, Weigand SD, et al. MRI and CSF biomarkers in normal, $\mathrm{MCl}$, and $\mathrm{AD}$ subjects: diagnostic discrimination and cognitive correlations. Neurology. 2009;73(4):287-93.

22. Vemuri $P$, Wiste HJ, Weigand SD, et al. Serial MRI and CSF biomarkers in normal aging, $\mathrm{MCl}$, and AD. Neurology. 2010;75(2):143-51.

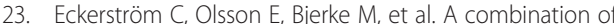
neuropsychological, neuroimaging, and cerebrospinal fluid markers predicts conversion from mild cognitive impairment to dementia. J Alzheimers Dis. 2013;36(3):421-31.

24. Shaffer JL, Petrella JR, Sheldon FC, et al. Predicting cognitive decline in subjects at risk for Alzheimer disease by using combined cerebrospinal fluid MR imaging, and PET biomarkers. Radiology. 2013;266(2):583-91.

25. Landau SM, Harvey D, Madison CM, et al. Comparing predictors of conversion and decline in mild cognitive impairment. Neurology. 2010;75(3):230-8.

26. van Rossum IA, Vos SJ, Burns $L$, et al. Injury markers predict time to dementia in subjects with $\mathrm{MCl}$ and amyloid pathology. Neurology. 2012; 79(17):1809-16.

27. Karow DS, McEvoy LK, Fennema-Notestine C, et al. Relative capability of MR imaging and FDG PET to depict changes associated with prodromal and early Alzheimer disease. Radiology. 2010;256(3):932-42.

28. Kantarci K, Weigand SD, Przybelski SA, et al. Risk of dementia in MCl: combined effect of cerebrovascular disease, volumetric MRI, and $1 \mathrm{H}$ MRS. Neurology. 2009;72(17):1519-25.

29. Caselli RJ, Jack Jr CR, Petersen RC, et al. Asymmetric cortical degenerative syndromes: clinical and radiologic correlations. Neurology. 1992;42:1462-8.

30. Varma AR, Adams W, Lloyd JJ, et al. Diagnostic patterns of regional atrophy on MRI and regional cerebral blood flow change on SPECT in young onset patients with Alzheimer's disease, frontotemporal dementia and vascular dementia. Acta Neurol Scand. 2002;105(4):261-9.

31. Niemantsverdriet $E_{1}$ Feyen BF, Le Bastard $N$, et al. Overdiagnosing vascular dementia using structural brain imaging for dementia work-up. J Alzheimers Dis. 2015:45(4):1039-43.

32. Chu WZ, Gong L, Xu YQ, et al. Apolipoprotein E gene variants of Alzheimer's disease and vascular dementia patients in a community population of Nanking. Med Chem. 2014;10(8):783-8.

33. Smith CD, Johnson ES, Van Eldik LJ, et al. Peripheral (deep) but not periventricular MRI white matter hyperintensities are increased in clinical vascular dementia compared to Alzheimer's disease. Brain Behav. 2016;16: e00438. doi:10.1002/brb3.438.

34. Logue MW, Posner $\mathrm{H}$, Green RC, et al. Magnetic resonance imagingmeasured atrophy and its relationship to cognitive functioning in vascular dementia and Alzheimer's disease patients. Alzheimers Dement. 2011:7(5):493-500

35. Boutoleau-Bretonnière $C$, Lebouvier $T$, Delaroche $O$, et al. Value of neuropsychological testing, imaging, and CSF biomarkers for the differential diagnosis and prognosis of clinically ambiguous dementia. J Alzheimers Dis. 2012:28(2):323-36.

36. Racine AM, Adluru N, Alexander AL, et al. Associations between white matter microstructure and amyloid burden in preclinical Alzheimer's disease: A multimodal imaging investigation. Neuroimage Clin. 2014:4:604-14

37. Dyrba M, Barkhof F, Fellgiebel A, et al. Predicting Prodromal Alzheimer's disease in subjects with Mild Cognitive Impairment using machine learning classification of multimodal multicenter diffusion-tensor and magnetic resonance imaging data. J Neuroimaging. 2015;25(5):738-47.

38. Balachandar R, John JP, Saini J, et al. A study of structural and functional connectivity in early Alzheimer's disease using rest fMRI and diffusion tensor imaging. Int J Geriatr Psychiatry. 2015:30(5):497-4.

39. Lee DY, Fletcher $E$, Martinez $O$, et al. Regional pattern of white matter microstructural changes in normal aging, MCl, and AD. Neurology. 2009; 73(21):1722-8 
40. Zarei M, Damoiseaux JS, Morgese C, et al. Regional white matter integrity differentiates between vascular dementia and Alzheimer disease. Stroke. 2009;40(3):773-9

41. Stahl R, Dietrich O, Teipel SJ, et al. White matter damage in Alzheimer disease and mild cognitive impairment: assessment with diffusion-tensor MR imaging and parallel imaging techniques. Radiology. 2007;243(2):483-92.

42. Meier IB, Manly JJ, Provenzano FA, et al. White matter predictors of cognitive functioning in older adults. J Int Neuropsychol Soc. 2012;18(3):414-27.

43. Smith EE, Egorova $\mathrm{S}$, Blacker D, et al. Magnetic resonance imaging white matter hyperintensities and brain volume in the prediction of mild cognitive impairment and dementia. Arch Neurol. 2008;65(1):94-100.

44. Yoshita M, Fletcher E, Harvey D, et al. Extent and distribution of white matter hyperintensities in normal aging, $\mathrm{MCl}$, and AD. Neurology. 2006; 67(12):2192-8

45. Prescott JW, Guidon A, Doraiswamy PM, et al. The Alzheimer structural connectome: changes in cortical network topology with increased amyloid plaque burden. Radiology. 2014;273(1):175-84.

46. Teipel SJ, Grothe MJ, Filippi M, et al. Fractional anisotropy changes in Alzheimer's disease depend on the underlying fiber tract architecture: a multiparametric DTI study using joint independent component analysis. J Alzheimers Dis. 2014;41(1):69-83.

47. Zhang $\mathrm{Y}$, Schuff $\mathrm{N}$, Jahng $\mathrm{GH}$, et al. Diffusion tensor imaging of cingulum fibers in mild cognitive impairment and Alzheimer disease. Neurology. 2007; 68(1):13-9.

48. Kantarci K, Jack Jr CR, Xu YC, et al. Mild cognitive impairment and Alzheimer disease: regional diffusivity of water. Radiology. 2001;219(1):101-7.

49. Takahashi R, Ishii K, Senda M, et al. Equal sensitivity of early and late scans after injection of FDG for the detection of Alzheimer pattern: an analysis of 3D PET data from J-ADNI, a multi-center study. Ann Nucl Med. 2013;27(5): 452-9.

50. Mendez MF, Ottowitz W, Brown CV, et al. Dementia with leukoaraiosis: clinical differentiation by temporoparietal hypometabolism on (18)FDGPETimaging. Dement Geriatr Cogn Disord. 1999;10(6):518-25.

51. Mosconi L, De Santi S, Brys M, et al. Hypometabolism and altered cerebrospinal fluid markers in normal apolipoprotein E E4 carriers with subjective memory complaints. Biol Psychiatry. 2008:63(6):609-18.

52. Mosconi L, Mistur R, Switalski R, et al. Declining brain glucose metabolism in normal individuals with a maternal history of Alzheimer disease. Neurology. 2009;72(6):513-20

53. Chételat $G$, Desgranges $B$, de la Sayette V. Mild cognitive impairment: can FDG-PET predict who is to rapidly convert to Alzheimer's disease? Neurology. 2003;60:1374-7.

54. Klupp E, Förster S, Grimmer T, et al. In Alzheimer's disease, hypometabolism in low-amyloid brain regions may be a functional consequence of pathologies in connected brain regions. Brain Connect. 2014;4(5):371-83.

55. Kim MJ, Lee KM, Son YD, et al. Increased basal forebrain metabolism in mild cognitive impairment: an evidence for brain reserve in incipient dementia. J Alzheimers Dis. 2012;32(4):927-38

56. Landau SM, Harvey D, Madison CM, et al. Associations between cognitive, functional, and FDG-PET measures of decline in AD and $\mathrm{MCl}$. Neurobiol Aging. 2011;32(7):1207-18.

57. Shokouhi S, Claassen D, Kang H, et al. Longitudinal progression of cognitive decline correlates with changes in the spatial pattern of brain 18 F-FDG PET. J Nucl Med. 2013;54(9):1564-9.

58. Ossenkoppele R, Tolboom N, Foster-Dingley JC, et al. Longitudinal imaging of Alzheimer pathology using [11C]PIB, [18 F]FDDNP and [18 F]FDG PET. Eur J Nucl Med Mol Imaging. 2012;39(6):990-1000

59. Chen Y, Wolk DA, Reddin JS, et al. Voxel-level comparison of arterial spinlabeled perfusion MRI and FDG-PET in Alzheimer disease. Neurology. 2011; 77(22):1977-85.

60. Albin RL, Koeppe RA, Burke JF, et al. Comparing fludeoxyglucose F18-PET assessment of regional cerebral glucose metabolism and [11C]dihydrotetrabenazine-PET in evaluation of early dementia and mild cognitive impairment. Arch Neurol. 2010;67(4):440-6.

61. Ji B, Maeda J, Sawada M, et al. Imaging of peripheral benzodiazepine receptor expression as biomarkers of detrimental versus beneficial glial responses in mouse models of Alzheimer's and other CNS pathologies. J Neurosci. 2008;28(47):12255-67.

62. Zimmer ER, Leuzy A, Benedet AL, et al. Tracking neuroinflammation in Alzheimer's disease: the role of positron emission tomography imaging. J Neuroinflammation. 2014;11:120.
63. Carter SF, Scholl M, Almkvist O, et al. Evidence for astrocytosis in prodromal Alzheimer disease provided by 11C-deuterium-L-deprenyl: a multitracer PET paradigm combining 11C-Pittsburgh compound B and 18 F-FDG. J Nucl Med. 2012:53:37-46.

64. Balsinde J, Winstead MV, Dennis EA. Phospholipase A(2) regulation of arachidonic acid mobilization. FEBS Lett. 2002;531:2-6.

65. Mizrahi R, Rusjan PM, Kennedy J, et al. Translocator protein (18 kDa) polymorphism (rs6971) explains in-vivo brain binding affinity of the PET radioligand [18 F]-FEPPA. J Cereb Blood Flow Metab. 2012;32:968-72.

66. Rosenberg PB, Wong DF, Edell SL, et al. Cognition and amyloid load in Alzheimer disease imaged with florbetapir F18(AV-45) positron emission tomography. Am J Geriatr Psychiatry. 2013;21(3):272-8.

67. Jagust WJ, Bandy D, Chen K, et al. The Alzheimer's Disease Neuroimaging Initiative positron emission tomography core. Alzheimers Dement. 2010;6(3): 221-9.

68. Klunk WE, Engler H, Nordberg A, et al. Imaging brain amyloid in Alzheimer's disease with Pittsburgh Compound-B. Ann Neurol. 2004;55:306-19.

69. Rowe CC, $\mathrm{Ng} \mathrm{S}$, Ackermann $U$, et al. Imaging beta-amyloid burden in aging and dementia. Neurology. 2007;68:1718-25.

70. Doraiswamy PM, Sperling RA, Johnson K, et al. Florbetapir F 18 amyloid PET and 36-month cognitive decline: a prospective multicenter study. Mol Psychiatry. 2014;19(9):1044-51.

71. Doraiswamy PM, Sperling RA, Coleman RE, et al. Amyloid- $\beta$ assessed by florbetapir F 18 PET and 18-month cognitive decline: a multicenter study. Neurology. 2012;79(16):1636-44.

72. Okello A, Koivunen J, Edison P, et al. Conversion of amyloid positive and negative $\mathrm{MCl}$ to AD over 3 years: an 11C-PIB PET study. Neurology. 2009; 73(10):754-60.

73. Johnson KA, Sperling RA, Gidicsin CM, et al. Florbetapir (F18-AV-45) PET to assess amyloid burden in Alzheimer's disease dementia, mild cognitive impairment, and normal aging. Alzheimers Dement. 2013;9(5 Suppl):S72-83.

74. Camus V, Payoux P, Barré L, et al. Using PET with 18 F-AV-45 (florbetapir) to quantify brain amyloid load in a clinical environment. Eur J Nucl Med Mol Imaging. 2012;39(4):621-31.

75. Furst AJ, Rabinovici GD, Rostomian AH, et al. Cognition, glucose metabolism and amyloid burden in Alzheimer's disease. Neurobiol Aging. 2012;33(2): 215-25.

76. Ong $K T$, Villemagne $V L$, Bahar-Fuchs $A$, et al. $A \beta$ imaging with $18 \mathrm{~F}$ florbetaben in prodromal Alzheimer's disease: a prospective outcome study. J Neurol Neurosurg Psychiatry. 2015;86(4):431-6.

77. Landau SM, Lu M, Joshi AD, et al. Comparing positron emission tomography imaging and cerebrospinal fluid measurements of $\beta$-amyloid. Ann Neurol. 2013;74(6):826-36.

78. Small GW, Siddarth P, Kepe $V$, et al. Prediction of cognitive decline by positron emission tomography of brain amyloid and tau. Arch Neurol. 2012; 69(2):215-22.

79. Murray ME, Graff-Radford NR, Ross OA, et al. Neuropathologically defined subtypes of Alzheimer's disease with distinct clinical characteristics: a retrospective study. Lancet Neurol. 2011;10(9):785-96.

80. Braak H, Braak E. Development of Alzheimer-related neurofibrillary changes in the neocortex inversely recapitulates cortical myelogenesis. Acta Neuropathol. 1996;92(2):197-201.

81. Chien DT, Bahri S, Szardenings AK, et al. Early clinical PET imaging results with the novel PHF-tau radioligand [F-18]-T807. J Alzheimers Dis. 2013;34(2):457-68.

82. Chien DT, Szardenings AK, Bahri S, et al. Early clinical PET imaging results with the novel PHF-tau radioligand [F18]-T808. J Alzheimers Dis. 2014;38(1):171-84

83. Maruyama M, Shimada $H$, Suhara $T$, et al. Imaging of tau pathology in a tauopathy mouse model and in Alzheimer patients compared to normal controls. Neuron. 2013;79(6):1094-108.

84. Greicius MD, Srivastava G, Reiss AL, et al. Default-mode network activity distinguishes Alzheimer's disease from healthy aging: evidence from functional MRI. Proc Natl Acad Sci U S A. 2004;101(13):4637-42.

85. Pihlajamaki M, Jauhiainen AM, Soininen $H$. Structural and functional MRI in mild cognitive impairment. Curr Alzheimer Res. 2009;6(2):179-85.

86. Jin M, Pelak VS, Cordes D. Aberrant default mode network in subjects with amnestic mild cognitive impairment using resting-state functional MRI. Magn Reson Imaging. 2012;30(1):48-61.

87. Sperling RA, Dickerson BC, Pihlajamaki M, et al. Functional alterations in memory networks in early Alzheimer's disease. Neuromolecular Med. 2010; 12(1):27-43 
88. Gomez-Ramirez J, Wu J. Network-based biomarkers in Alzheimer's disease: review and future directions. Front Aging Neurosci. 2014;6:12.

89. Wang $Y$, Risacher $S$, West J, et al. Altered default mode network connectivity in older adults with cognitive complaints and amnestic mild cognitive impairment. J Alzheimers Dis. 2013;35:751-60.

90. Zhu DC, Majumdar S, Korolev IO, et al. Alzheimer's disease and amnestic mild cognitive impairment weaken connections within the default-mode network: a multi-modal imaging study. J Alzheimers Dis. 2013;34(4):969-84.

91. Petrella JR, Sheldon FC, Prince SE, et al. Default mode network connectivity in stable vs progressive mild cognitive impairment. Neurology. 2011;76(6): 511-7.

92. Flicker L. Modifiable lifestyle risk factors for Alzheimer's disease. J Alzheimers Dis. 2010;20:803-11.

93. Dickerson BC, Salat DH, Greve DN, et al. Increased hippocampal activation in mild cognitive impairment compared to normal aging and $A D$. Neurology. 2005;65(3):404-11.

94. O'Brien JL, O'Keefe KM, LaViolette PS, et al. Longitudinal fMRI in elderly reveals loss of hippocampal activation with clinical decline. Neurology. 2010; 74(24):1969-76.

95. Mondadori CR, Buchmann A, Mustovic H, et al. Enhanced brain activity may precede the diagnosis of Alzheimer's disease by 30 years. Brain. 2006;129(Pt 11):2908-22.

96. Bero AW, Yan $\mathrm{P}$, Roh $\mathrm{JH}$, et al. Neuronal activity regulates the regional vulnerability to amyloid- $\beta$ deposition. Nat Neurosci. 2011;14(6):750-6.

97. Kim J, Basak JM, Holtzman DM. The role of apolipoprotein E in Alzheimer's disease. Neuron. 2009;63:287-303.

98. Lacalle-Aurioles M, Mateos-Pérez JM, Guzmán-De-Villoria JA, et al. Cerebral blood flow is an earlier indicator of perfusion abnormalities than cerebral blood volume in Alzheimer's disease. J Cereb Blood Flow Metab. 2014;34(4): 654-9.

99. Huang C, Wahlund LO, Svensson L, et al. Cingulate cortex hypoperfusion predicts Alzheimer's disease in mild cognitive impairment. BMC Neurol. 2002;2:9.

100. Encinas M, De Juan R, Marcos A, et al. Regional cerebral blood flow assessed with $99 \mathrm{mTC}$-ECD SPET as a marker of progression of mild cognitive impairment to Alzheimer's disease. Eur J Nucl Med Mol Imaging. 2003; 30(11):1473-80.

101. Habert MO, Horn JF, Sarazin M, et al. Brain perfusion SPECT with an automated quantitative tool can identify prodromal Alzheimer's disease among patients with mild cognitive impairment. Neurobiol Aging. 2011; $32(1): 15-23$

102. Devanand DP, Van Heertum RL, Kegeles LS, et al. (99 m)Tc hexamethylpropylene-aminoxime single-photon emission computed tomography prediction of conversion from mild cognitive impairment to Alzheimer disease. Am J Geriatr Psychiatry. 2010;18(11):959-72.

103. Chen YJ, Deutsch G, Satya R, et al. A semi-quantitative method for correlating brain disease groups with normal controls using SPECT: Alzheimer's disease versus vascular dementia. Comput Med Imaging Graph. 2013:37(1):40-7

104. Borroni B, Anchisi D, Paghera B, et al. Combined 99mTc-ECD SPECT and neuropsychological studies in $\mathrm{MCl}$ for the assessment of conversion to $\mathrm{AD}$. Neurobiol Aging. 2006;27(1):24-31.

105. Gao YZ, Zhang JJ, Liu H, et al. Regional cerebral blood flow and cerebrovascular reactivity in Alzheimer's disease and vascular dementia assessed by arterial spinlabeling magnetic resonance imaging. Curr Neurovasc Res. 2013;10(1):49-53.

106. Hanyu $H$, Kume $K$, Sato $T$, et al. Regional differences in cortical benzodiazepine receptors of Alzheimer, vascular, and mixed dementiapatients. J Neurol Sci. 2012;323(1-2):71-6.

107. Yoshikawa T, Murase $\mathrm{K}, \mathrm{Oku} \mathrm{N}$, et al. Heterogeneity of cerebral blood flow in Alzheimer disease and vascular dementia. AJNR Am J Neuroradiol. 2003; 24(7):1341-7.

108. Sperling RA, Aisen PS, Beckett LA, et al. Toward defining the preclinical stages of Alzheimer's disease: recommendations from the National Institute on Aging-Alzheimer's Association workgroups on diagnostic guidelines for Alzheimer's disease. Alzheimers Dement. 2011;7(3):280-92.

109. Price $J$, Morris JC. Tangles and plaques in nondementted aging and "preclinical" Alzheimer's disease. Ann Neurol. 1999;45:358-68.

110. Montagne A, Barnes SR, Sweeney MD, et al. Blood-brain barrier breakdown in the aging human hippocampus. Neuron. 2015;85(2):296-302.
111. Jack CRJ, Lowe VJ, Senjem ML, et al. 11C PiB and structural MRI provide complementary information in imaging of Alzheimer's disease and amnestic mild cognitive impairment. Brain. 2008;131(Pt 3):665-80.

\section{Submit your next manuscript to BioMed Central and we will help you at every step:}

- We accept pre-submission inquiries

- Our selector tool helps you to find the most relevant journal

- We provide round the clock customer support

- Convenient online submission

- Thorough peer review

- Inclusion in PubMed and all major indexing services

- Maximum visibility for your research

Submit your manuscript at www.biomedcentral.com/submit 